\title{
Some Remarks on the Stability of Production Networks
}

\author{
Bernd Scholz-Reiter ${ }^{1}$, Fabian Wirth ${ }^{2}$, Michael Freitag ${ }^{1}$, Sergey \\ Dashkovskiy $^{2}$, Thomas Jagalski ${ }^{1}$, Christoph de Beer ${ }^{1}$, and Björn Rüffer ${ }^{2}$ \\ 1 University of Bremen, Department of Planning and Control of Production \\ Systems, Germany \{bsr,fmt, jag, ber $\} @ b i b a . u n i-b r e m e n . d e$ \\ 2 University of Bremen, Zentrum für Technomathematik, Germany \\ \{fabian, dsn, rueffer\}@math.uni-bremen.de
}

Summary. The increasing complexity of production and logistics networks and the requirement of higher flexibility lead to a change of paradigm in control: Autonomously controlled systems where decisions are taken by parts or goods themselves become more attractive. The question of stability is an important issue for the dynamics of such systems. In this paper we are going to touch this question for a special production network with autonomous control. The stability region for a corresponding fluid model is found empirically. We point out that further mathematical investigations have to be undertaken to develop some stability criteria for autonomous systems.

\section{Introduction}

In view of modern information and communication technologies and the dynamics and complexity of production and logistics networks, the idea to employ decentralized autonomous control, i.e., to design a network as interconnected autonomous units able to make decisions themselves, seems to be a new paradigm in logistics due to its flexibility and robustness [1], [2], [3], [4], [5]. Networks with centralized control commonly used in past decades are well studied in the sense that there are different models like queuing, fluid and discrete models proposed. These models allow to predict the behavior of a system, its efficiency and provide the designer with criteria of stability cf. [6], [7]. In this paper we concentrate on the stability properties of production networks with autonomous control. We consider the same production scenario as in [3] and [4] and state a fluid model for it. With help of simulation we find the stability area of parameters. In Section 2 we briefly describe the model. In the Section 3 we introduce the notion of stability and quote some known results. Section 4 contains simulation results. We collect some conclusions in Section 5 . 


\section{Model Description}

The considered production network is a dynamic flow-line manufacturing system. It consists of $n$ parallel production lines each with $m$ machines $M_{i j}$ and an input buffer $B_{i j}$ in front of each machine (see Fig. 1). Every line processes a certain kind of product $1,2, \ldots, K$ by $m$ job steps. The raw materials for each product enter the system via sources; the final products leave the system via drains. The production lines are coupled at every stage and every line is able to process every type of product within a certain stage. The decision about changing the line is made as an autonomous decision by the part itself on the basis of local information about buffer

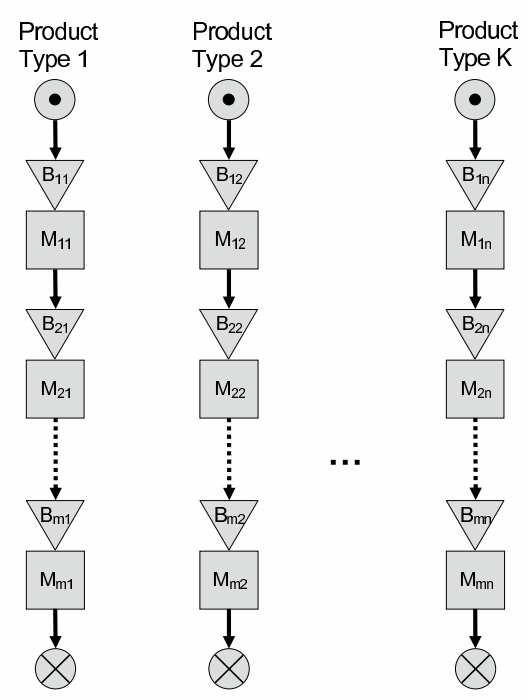
levels and expected waiting times

Fig. 1. $m \times n$ machines production network until processing.

To handle the complexity of the production network, the described scenario is reduced to $3 \times 3$ machines, i.e., three production lines each with three stages. The parts are autonomous in their decision which machine to choose. They take into account the fact that the processing times are higher on foreign lines than on their associated production line. At each production stage the parts compare the processing times of the parts in the buffers and their own processing time on the respective machine and choose the machine with the minimal time for being processed. Table 1 shows the processing times and the resulting processing rates for the three different product types on the three production lines.

Table 1. Processing times and resulting processing rates of the $3 \times 3$ machine model

\begin{tabular}{|c|c|c|c|}
\hline & \multicolumn{3}{|c|}{$\begin{array}{l}\text { Processing times [h:min]/ Processing } \\
\text { rates [parts/hour] at production line } \mathrm{j}\end{array}$} \\
\hline Part Type 1 & $2: 00 / 0.5$ & $2: 30 / 0.4$ & $3: 00 / 0.33$ \\
\hline Part Type 2 & $3: 00 / 0.33$ & $2: 00 / 0.5$ & $2: 30 / 0.4$ \\
\hline Part Type 3 & $2: 30 / 0.4$ & $3: 00 / 0.33$ & $2: 00 / 0.5$ \\
\hline
\end{tabular}

To analyse the system's behaviour at varying demand and workload fluctuations, an arrival function $\lambda(t)$ is defined and set as a sine function: 


$$
\lambda(t)=\lambda_{m}+\alpha \times \sin (t+\varphi)
$$

Here, $\lambda_{m}$ is the mean arrival rate, $\alpha$ is the amplitude of the sine function, and $\varphi$ indicates a phase shift. The arrival functions for the three product types 1,2 and 3 are identical except for the phase shift of $1 / 3$ period. This phase shift is chosen to simulate a seasonal varying demand for the three different products.

\section{The Notion of Stability}

There are several notions of stability for different models in the literature, see [7] for Harris recurrence of queuing networks, [6] for weak and strong stability of fluid models, [5] for Input-to-State stability in control systems. Roughly speaking this properties mean that the state of the system (or length of the queues) remains bounded if the external input to the system is bounded.

Let us consider a queuing network with $K$ classes of customers processed with service times $m_{k}, k=1, \ldots, K$; routing matrix $P$, external arrival rates $\lambda=\left(\lambda_{1}, \ldots, \lambda_{K}\right)^{\prime}$ and let $C_{i}$ be the set of classes processed on the server $i, \quad i=1, \ldots, I$. The effective arrival rate $\lambda_{\mathrm{eff}, k}$ to the class $k$ is given by $\lambda_{\text {eff }}=\left(I-P^{\prime}\right)^{-1} \lambda$. Then for some special networks and some special service disciplines the condition

$$
\rho_{i}:=\sum_{k \in C_{i}} \lambda_{\mathrm{eff}, k} m_{k}<1
$$

was found to be a sufficient for stability of the corresponding network. However changing the discipline may cause instability, see [8].

It is known that stability of fluid limit guarantees the stability of the corresponding queuing network. However if the fluid limit is unstable one can conclude nothing about the stability of queuing network. In the following we describe some simulation results concerning stability of the described $3 \times 3$ machines model. The known criteria are not applicable for this model because of the autonomous routing by the parts themselves.

\section{Simulation Results}

\subsection{Stability Region Using Fluid Models}

The fluid model for the $3 \times 3$ machines model is given by the following set of equations: 


$$
\begin{gathered}
Q_{i j k}(t)=Q_{i j k}(0)+\int_{0}^{t} \lambda_{i j k}(s)-\mu_{i j k} \dot{T}_{i j k}(s) d s, \\
\lambda_{i k} \equiv \sum_{j=1}^{3} \lambda_{i j k}, \\
\lambda_{i j k}(t)=\lambda_{i k}(t) \times \mathbb{1}_{\left\{W_{i j}(t)+\frac{\lambda_{i k}(t) \times d t}{\mu_{i j k}} \leq \min _{l \neq j}\left\{W_{i l}(t)+\frac{\lambda_{i k}(t) \times d t}{\mu_{i l k}}\right\}\right\}}, \\
\lambda_{i+1, k}(t)=\sum_{j=1}^{3} \mu_{i j k} \dot{T}_{i j k}(t), \text { for } i=1,2, \\
\dot{T}_{i j k}(t)= \begin{cases}0, & \text { if } Q_{i j k}(t)=0, \\
\sum_{l=1}^{3} Q_{i j l}(t) & \text { else },\end{cases} \\
W_{i j}(t)=\sum_{k=1}^{k} \frac{Q_{i j k}(t)}{\mu_{i j k}} .
\end{gathered}
$$

Here $i$ and $j$ denote the row and column of each machine in the network, $k$ refers to the product type, $Q$ is the queue length in fractions of products, $W$ the amount of work in the queue, $\mu$ is constant and describes the maximal possible service rates, $T$ is the cumulative allocation time per machine and product type, it increases whenever the respective server spends time serving the corresponding type of part. $\lambda_{i k}$ denotes the arrival rate of part $k$ in row $i$, which then is directed to exactly one machine.

Fluid limits do not capture individual parts, they can be seen as a macroscopic view of the process, such that all external arrival rates become their averages. The autonomous routing is captured in (5) under the restriction of (4), that is, the input rate can only turn to one machine at the time. By the macroscopic perspective we may assume (7), since in the FIFO queues of each server and by the intelligent routing algorithm, the parts are assumed to be equally distributed, so that each server spends service time for each class of products as it relates to the total queue length in front of the machine.

Using standard discrete time numerical methods, we calculate estimates on the stability region of the fluid limit, which in turn is a subset of the stability region of the discrete event system, see Fig. 2 lower curve (solid line). Here we used an exemplary arrival rate of 0.4 parts / hour (as in $[3,4]$ ) for product type 3 and varied the arrival rates for types 1 and 2 .

\subsection{Stability Region Using Discrete Event Simulation}

To analyse the stable parameter region using the discrete event simulation analogue to the continuous method the arrival rate for part type 3 is set as constant, in this case $\lambda_{3}=0.4$ parts / hour. The other two arrival rates are still sine functions with an amplitude of $\alpha=0.15$ parts / hour. The mean of the sine curves are independently varied, i.e., one of the mean arrival rates is 
Fig. 2. Subset of the stability region of the $3 \times 3$ machine model with a given arrival rate of 0.4 parts of type 3 per hour

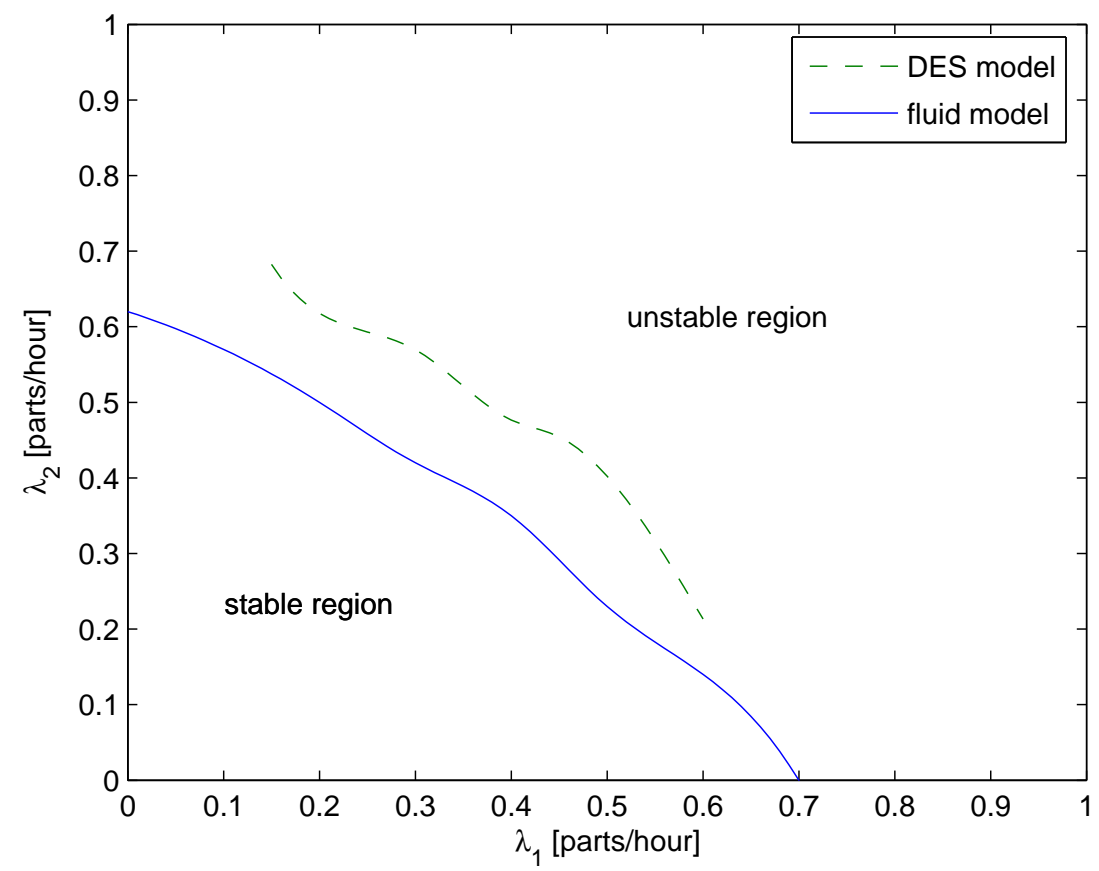

held constant while the other is increased unless the buffer levels begin to rise to infinity. The maximum mean arrival rate before the buffer levels begin to rise to infinity is called the critical rate. The result of this stability analysis is shown in Fig. 2 upper curve (dashed line).

The minimal mean arrival rates $\lambda_{1}$ and $\lambda_{2}$ are 0.15 parts / hour because the amplitude is $\alpha=0.15$ parts / hour and no negative arrival rates are allowed.

\section{Conclusions}

Using the fluid model, a general statement about stability of the $3 \times 3$ machines production network could be derived, i.e., a stability margin (cf. lower curve (solid line) in Fig. 2) independent from a specific arrival function $\lambda(t)$. The DES model, on the other hand, provided a stability margin (cf. upper curve (dashed line) in Fig. 2) for a specific arrival function $\lambda(t)$. Due to the particular parameter settings, the stability margin of the DES model is above the margin of the fluid model. 
The stability of the network depends on the specific parameters for the arrival rates and service rates, but a definitely stable parameter region could be found by the fluid model.

\section{Acknowledgments}

This research is funded by the German Research Foundation (DFG) as part of the Collaborative Research Centre 637 "Autonomous Cooperating Logistic Processes: A Paradigm Shift and its Limitations" (SFB 637).

\section{References}

1. Scholz-Reiter B, Freitag M, Windt K (2004) Autonomous logistic processes. In: Proceedings of the 37th CIRP International Seminar on Manufacturing Systems 357-362

2. Dashkovskiy S, Wirth F, Jagalski T (2004) Autonomous control of Shop Floor Logistics: Analytic models. In: Proceedings of the IFAC Conference on Manufacturing, Modelling, Management and Control. On CD-ROM

3. Scholz-Reiter B, Freitag M, de Beer C, Jagalski T (2005) Modelling dynamics of autonomous logistic processes: Discrete-event versus continuous approaches. Annals of the CIRP 55:413-416

4. Scholz-Reiter B, Freitag M, de Beer C, Jagalski T (2005) Modelling and analysis of autonomous shop floor control. In: Proceedings of the 38th CIRP International Seminar on Manufacturing Systems. On CD-ROM

5. Dashkovskiy S, Rüffer B, Wirth F (2005) An ISS Small-Gain Theorem for General Networks. In: Proceedings of the Joint 44th IEEE Conference on Decision and Control and European Control Conference (CDC-ECC'05). To appear.

6. Chen H (1995) Fluid approximations and stability of multiclass queueing networks: work-conserving disciplines. In: Annals of Applied Probability 5:637-665

7. Dai JG (1995) On positive Harris recurrence of multiclass queueing networks: a unified approach via fluid limit models. In: Annals of Applied Probability 5:4977

8. Bramson M (1994) Instability of FIFO queueing networks. Annals of Applied Probability 4:414-431 